\title{
Social contexts modulate neural responses in the processing of others' pain: An event-related potential study
}

\author{
Fang Cui ${ }^{1,2} \cdot$ Xiangru Zhu ${ }^{3}$ Yuejia Luo ${ }^{1,2,4}$
}

Published online: 26 May 2017

(C) Psychonomic Society, Inc. 2017

\begin{abstract}
Two hypotheses have been proposed regarding the response that is triggered by observing others' pain: the "empathizing hypothesis" and the "threat value of pain hypothesis." The former suggests that observing others' pain triggers an empathic response. The latter suggests that it activates the threat-detection system. In the present study, participants were instructed to observe pictures that showed an anonymous hand or foot in a painful or non-painful situation in a threatening or friendly social context. Event-related potentials were recorded when the participants passively observed these pictures in different contexts. We observed an interaction between context and picture in the early automatic N1 component, in which the painful pictures elicited a larger amplitude than the non-painful pictures only in the threatening context and not in the friendly context. We also observed an interaction between context and picture in the late P3 component, in which the painful pictures elicited a larger amplitude than the non-painful pictures only in the friendly context and not in the threatening context. These results indicate that specific social contexts can modulate the neural responses to observing others' pain. The "empathic hypothesis" and "threat value of pain hypothesis" are not mutually exclusive and do not contradict each other but rather work in different temporal stages.
\end{abstract}

Fang Cui

cuifang0826@gmail.com

1 Shenzhen Key Laboratory of Affective and Social Cognitive Science, Shenzhen University, Nanhai Ave 3688, Shenzhen, Guangdong, China 518060

2 College of Psychology and Sociology, Shenzhen University, Shenzhen, China

3 Institute of psychology and behavior, Henan University, Kaifeng, China

4 Shenzhen Institute of Neuroscience, Shenzhen, China
Keywords Other's pain - ERPs - Threatening context · Friendly context $\cdot \mathrm{N} 1 \cdot \mathrm{P} 3$

\section{Introduction}

Observing the pain of others is a very salient stimulus. Eventrelated potential (ERP) studies demonstrate more positivity in the N1, N2 and P3 components in response to painful stimuli than to non-painful stimuli (Han, Fan, \& Mao, 2008a; Ibanez et al., 2011; Lyu, Meng, \& Jackson, 2014; Meng et al., 2012). Functional magnetic resonance imaging studies suggest that observing others who are in painful situations triggers stronger activation of a larger set of brain regions, including the anterior insula, anterior cingulate cortex, and amygdala (Keysers, Meffert, \& Gazzola, 2014; Keysers, Kaas, \& Gazzola, 2010; Lamm, Decety, \& Singer, 2011; Rizzolatti \& Sinigaglia, 2010), compared with observing others who are in nonpainful situations.

Two hypotheses have been proposed regarding the responses that are triggered by observing others' pain: the "empathizing hypothesis" and the "threat value of pain hypothesis" (TVPH). The "empathizing hypothesis" suggests that observing others' pain triggers empathy for the others' pain. Empathy refers to the ability to share and understand others' emotions or feelings (Decety \& Lamm, 2006). Empathy relies on the integration of two components: an automatic early emotional contagion system and a more advanced cognitive system that allows self-regulation and elaboration of the situation (Decety \& Jackson, 2004; Mella, Studer, Gilet, \& Labouvie-Vief, 2012). From a temporal perspective, ERP studies support the two-component model, suggesting that empathizing with pain involves two corresponding temporal processes: early, automatic processing that results in emotion al contagion and affective sharing (reflected by a more 
positive early automatic component [EAC], such as N1 and $\mathrm{N} 2$, in response to painful stimuli compared with non-painful stimuli) and a later, cognitively controlled process that is subserved by the prefrontal cortex to regulate empathic responses and to make a clear distinction between the self and other (reflected by more positive amplitudes of centro-parietal P3 in response to painful stimuli compared with non-painful stimuli; Fan \& Han, 2008; Mella et al., 2012; Sessa, Meconi, Castelli, \& Dell'Acqua, 2014).

The TVPH suggests that both the early and the late processing of painful stimuli are associated with a potential threat, and the mere perception of others' pain does not automatically activate an empathic process. Rather, a threat-detection system appears to be activated first, with a possibly general aversive response in the observer, instead of an empathic response (Ibanez et al., 2011). However, compared with the "empathizing hypothesis," the TVPH is relatively new, and few studies can be found in the literature that have tested or verified it.

Social interactions, such as understanding others' feelings and emotions, naturally occur in context-rich settings that modulate all aspects of cognition, from basic perception to the interpersonal domains (Ibanez \& Manes, 2012). Empathy for pain in particular is a highly contextual phenomenon (Melloni, Lopez, \& Ibanez, 2014). Numerous studies have suggested that the neural response to others' pain is flexible and can be modulated by contextual factors (Akitsuki \& Decety, 2009; Decety, Echols, \& Correll, 2010; Cui et al., 2016 Cui, Ma, \& Luo, 2016; Gu \& Han, 2007; Lyu et al., 2014; Singer et al., 2004). A reasonable assumption is that the context, especially the social context, influences the way in which we process others' pain. However, the ways in which the social contexts modulate the processing of others' pain has not yet been explored.

When you hear a gunshot on the street, then seeing a person who is covered with blood may make you want to run away. In contrast, when you see the suffering of a child in a hospital, you may want to approach and comfort him. Under different social contexts, others' pain may signal danger or a call for help. In the present study, we explored the ways in which stimuli that show others' pain are processed in different social contexts. "Social context" refers to the immediate physical and social setting where people live or where something happens or develops (Barnett \& Casper, 2001). We manipulated the social context by presenting pictures of different facial expressions to the participants. Angry faces were used to induce a threatening context, and smiling faces were used to induce a friendly context; the faces have all been shown to be valid in the literature (Karos, Meulders, \& Vlaeyen, 2015).

A modified affective oddball paradigm was used to create the affective contexts. Using the affective oddball paradigm to trigger an affective context has also been shown to be valid (Cui, Zhu, Duan, \& Luo, 2015; Schupp et al., 2000). The oddball paradigm can enable the unpredictability of target stimuli. The unpredictability of stimuli that show others' pain can exclude confounding factors such as expectation and preparation. Two types of blocks were used: "threatening" block and "friendly" block. In the threatening blocks, angry faces were the standard stimuli, and pictures that showed others who were in painful or non-painful situations were the deviant stimuli. In the friendly blocks, smiling faces were the standard stimuli. We compared ERPs while the participants viewed the deviant pictures in the different blocks.

Our first hypothesis was that the participants would be more sensitive and alert to others' pain in the threatening context than in the friendly context in the early perceptual processing stage, reflected by EACs, such as N1 and N2. Such an effect would be better explained by the TVPH because others' pain does not trigger an empathic response but rather activates the danger detection system. One ERP study found that the threat detection system was enhanced when primed with information that was associated with others compared with information that was associated with one's own because the other represents a stronger threat than the self (Ibanez et al., 2011).Greater sensitivity to harm in the threatening context could be evolutionally beneficial because harm that affects others mostly is a danger signal to the self as well.

Our second hypothesis was that there is a weaker empathic response to others' pain in a threatening context than in a friendly context. One recent study found that the stress of being around strangers might be responsible for the absence of empathizing with the pain of strangers (Martin et al., 2015). This finding indicates that when our own safety is threatened, the stress prevents us from automatically empathizing with others' pain. In the design of the present study, this effect might be observed in later ERP components, such as P3. In the threatening context, one would retain the mental resources that are necessary to deal with potential dangers rather than process others' emotions and feelings. Moreover, if evidence is found to support both of these hypotheses, then this indicate that the empathizing hypothesis and TVPH might both be true and work in different temporal stages. The early perceptual processing stage can be considered a danger detection stage, which can be better explained by the TVPH. The observers would first judge whether others' pain signals a threat. The later, cognitive processing stage would be better explained by the empathizing hypothesis because the threat may prevent the observer from empathizing with others' pain.

\section{Methods}

\section{Participants}

Twenty-three right-handed participants with no history of neurological disorders, brain injuries, or developmental 
disabilities participated in the experiment. All of them had normal or corrected-to-normal vision. The research was approved by the Medical Ethics Committee of Shenzhen University Medical School according to the principles of the Declaration of Helsinki. All participants provided written informed consent. One participant's data were rejected due to intensive head movements during EEG recording $(27.4 \%$ bad epochs). Finally, 22 participants' data were included (nine male, age $23.95 \pm 0.45$ years (mean $\pm \mathrm{SE})$ ).

\section{Materials and design}

In the oddball paradigm, 60 pictures showing a person's hands/forearms/feet in painful or non-painful situations were used as deviant stimuli (30 painful and 30 non-painful); 180 pictures showing an angry face or a happy face were used as standard stimuli ( 90 angry faces and 90 happy faces). Genders of the faces were counterbalanced between the angry and happy faces.

The deviant stimuli used in the experiment were pictures showing a person's hands/forearms/feet in painful or nonpainful situations, which have been used in previous ERP studies (Meng et al., 2013). All the situations depicted in these pictures were ordinary events in daily life and all the events shown in the non-painful pictures corresponded to those in the painful pictures just without the nociceptive component (Fig. 1A).

The standard stimuli used in the experiment were selected from the native Chinese Facial Affective Picture System (CFAPS) including 90 happy faces and 90 angry faces (Fig. 1A). The recognition consistency and intensity of expressions were evaluated in the initial validation research (X, YX, Y, \& YJ, 2011). The recognition consistencies were 85.64 \pm 8.38 for happy expressions and $86.77 \pm 6.56$ for angry expressions. The intensities of happy and angry expressions were $6.35 \pm 0.78$ and $6.91 \pm 0.58$ (mean $\pm \mathrm{SD}$ ). No significant differences in recognition consistency or intensity were found between these two categories $(p>0.5)$. Male and female faces were represented equally. They were identical to each other in size, background, contrast grade, brightness, and other physical properties. All faces were gray-scale and were presented on a black background $\left(3.0^{\circ} \times 3.5^{\circ}\right.$ visual angle $)$. All of them had the same size, $9 \times 6.76 \mathrm{~cm}$ (width $\times$ height), with 100 pixels/ in.

During the experiment the participant sat in a dark and quiet room alone during the recording. A 15 -in. color monitor was placed in front of him/her. During the task, the pictures were presented in the center of a white background.

\section{Experimental procedures}

The study had a $2 \times 2$ within-subject design. The first factor was the "Context." There were two levels to this factor: the threatening context and the friendly context. The second factor was the pictures: the painful and the non-painful ones. A block design was used with each block contain 240 trials including $25 \%$ deviant stimuli (12.5\% painful and $12.5 \%$ non-painful pictures) and $75 \%$ standard stimuli. In the "Threatening context," all of the $75 \%$ standard stimuli were an angry face while in the "Friendly context", all of the $75 \%$ standard stimuli were a happy face. In each trial, a fixation was presented on a white screen for $500 \mathrm{~ms}$, followed by a deviant or a standard stimuli picture presented for $1,000 \mathrm{~ms}$. There was a blank interval presented for $400-700 \mathrm{~ms}$ between trials (Fig. 1B). There were two blocks of "threatening context" and two blocks of "friendly context." The order of the blocks was "ABBA," "BAAB," "ABAB," and "BABA," balanced among participants. There were 240 trials of each block and a maximum of 2 min rest between blocks. There were 960 trials in total. Each specific stimulus was repeated four times and the whole recording last approximately $55 \mathrm{~min}$.

In order to keep the participant's attention during the passive viewing task, the participant was told that questions would randomly appear after the deviant stimuli to ask if they saw a painful or a non-painful picture and they were to respond by pressing appropriate buttons. In each block, there were $25 \%$ (60) deviant stimuli and within them 10-20\% of the pictures were followed by a question randomly. The question that followed was presented immediately after the picture and disappeared until a response was given.

\section{EEG acquisition and analysis}

Electroencephalography (EEG) data were recorded from a 63electrode scalp cap using the 10-20 system (Brain Products, Munich, Germany). The channel TP10 was used as the reference during recording. Two electrodes were used to measure the electro-oculogram (EOG). EEG and EOG activity was amplified at $0.01-100 \mathrm{~Hz}$ band-passes and sampled at 500 Hz. All electrode impedances were maintained below $5 \mathrm{k} \Omega$. EEG data were pre-processed and analyzed using Matlab R2011b (MathWorks, Natick, MA, USA) and EEGLAB toolbox (Delorme \& Makeig, 2004). EEG data at each electrode were down-sampled to $250 \mathrm{~Hz}$. The data were then rereferenced to the common average, after which the signal passed through a $0.01-30 \mathrm{~Hz}$ band-pass filter. Time windows of $200 \mathrm{~ms}$ before and 1,000 ms after the onset of the picture were segmented from EEG. EOG artifacts were corrected using an independent component analysis (ICA) (Jung et al., 2001). Epochs with amplitude values exceeding $\pm 50 \mu \mathrm{V}$ at any electrode were excluded from the average.

Further statistical analysis was conducted in IBM SPSS Statistics 22 (IBM Corp., Armonk, NY, USA). Previous studies using similar stimuli suggest that EAC (mainly including $\mathrm{N} 1$ and N2) and P3 are particularly related to observing other's pain (Cui et al., 2016; Fan \& Han, 2008; Lyu et al., 


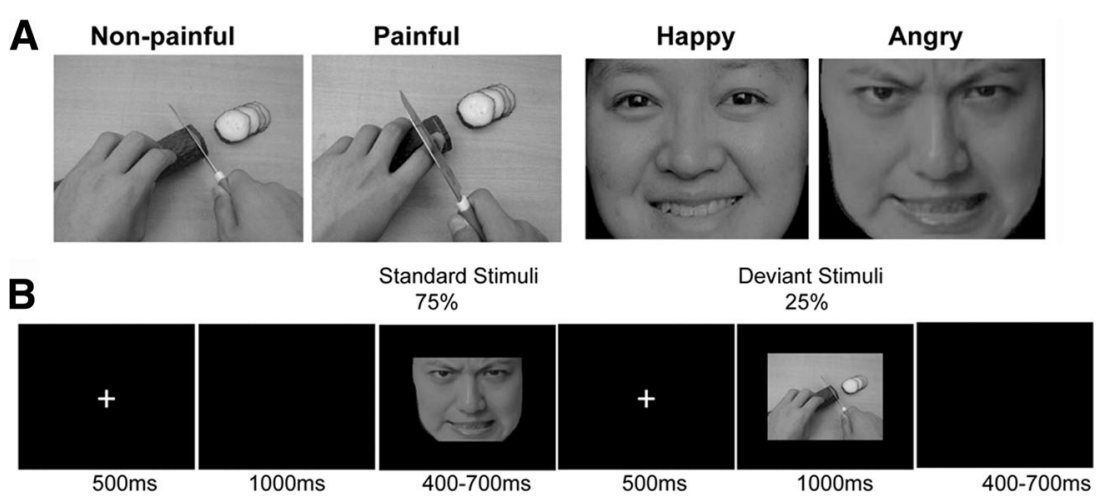

Fig. 1 (A) Examples of the deviant and standard pictures. (B) Example of one trial

2014; Meng et al., 2013; Meng et al., 2012). Analyses were conducted over the peak amplitude of the N1 and N2 components and the mean amplitudes of the P3 component. On the basis of the topographical distribution of grand-averaged ERP activity and previous studies, different sets of electrodes for each component were chosen. F3, Fz, F4, FC3, FCz, and FC3 electrode sites were selected for the analysis of N1 (110-160 $\mathrm{ms}$ ) and N2 (240-270 ms); FC3, FCz, FC4, C3, Cz, and C4 for the analysis of P2 (160-200 ms); CP3, CPz, CP4, P3, Pz, $\mathrm{P} 4, \mathrm{PO} 3, \mathrm{POz}$, and $\mathrm{PO} 4$ were selected for the analysis of $\mathrm{P} 3$ (350-550 ms). Repeated measures ANOVA (2 (Context: Threatening / Friendly) $\times 2$ (Picture: Painful / Non-Painful)) were performed for the mean amplitudes of all selected electrodes sites for each component. Degrees of freedom for $\mathrm{F}$ ratios were corrected according to the Greenhouse-Geisser method. Statistical differences were considered significant at $p<0.05$; post-hoc comparisons were Bonferroni-corrected at $p<0.05$.

\section{Results}

\section{Behavioral data}

The accuracy of the responses to questions in the threatening blocks and in the friendly blocks were $(98.37 \pm 0.14) \%$ and $(99.31 \pm 0.24) \%$ (mean $\pm \mathrm{SE})$, indicating that the participants did pay attention to the stimuli during the task. There were no significant differences between the two contexts in accuracy or reaction time $(\mathrm{RT})(p>0.5)$.

\section{Event-related potentials}

The total averaged ERPs for the pictures were computed separately for each condition. There are four conditions in total: the painful picture in the threatening context (T_P), the nonpainful pictures in the threatening context (T_NP), the painful picture in the friendly context (F_P), and the non-painful pictures in the friendly context (F_NP).
N1 A significant main effect of Picture was observed (F (1, $\left.21)=6.909, p=0.016, \eta_{\mathrm{p} 2}=0.248\right)$. The painful picture elicited more positive amplitudes than the non-painful pictures $(-1.045 \pm 0.120 \mu \mathrm{V}$ (mean $\pm \mathrm{SE}$ ) for painful stimuli and $-1.207 \pm 0.132 \mu \mathrm{V}$ for the non-painful ones). The main effect of Context was nonsignificantly observed $(\mathrm{F}(1,21)=1.117, p$ $\left.=0.302, \eta_{\mathrm{p} 2}=0.051\right)$. A significant interaction of Context $\times$ Picture $\left(\mathrm{F}(1,21)=4.918, p=0.038, \eta_{\mathrm{p} 2}=0.190\right)$ was observed. Pairwise comparison indicates that in the threatening context, the painful stimuli elicited a significantly more positive N1 than the non-painful stimuli $(-1.015 \pm 0.143 \mu \mathrm{V}$ for painful stimuli and $-1.354 \pm 0.167 \mu \mathrm{V}$ for non-painful stimuli, $p<0.001)$. Meanwhile, there was no significant difference between painful and non-painful stimuli on N1 in the friendly context on any electrodes site $(-1.074 \pm 0.130 \mu \mathrm{V}$ for painful stimuli and $-1.060 \pm 0.131 \mu \mathrm{V}$ for non-painful stimuli, $p=$ 0.904) (Fig. 2A and Fig. 3).

P2 The main effect of Picture $(\mathrm{F}(1,21)=0.289, p=0.596$, $\left.\eta_{\mathrm{p} 2}=0.014\right)$, main effect of Context $(\mathrm{F}(1,21)=0.024, p=$ $\left.0.879, \eta_{\mathrm{p} 2}=0.001\right)$, and the interaction of Context $\times$ Picture $(\mathrm{F}$ $\left.(1,21)=0.307, p=0.586, \eta_{\mathrm{p} 2}=0.014\right)$ were not significant.

N2 We observed a significant main effect of Picture $(\mathrm{F}(1,21)$ $=10.686, p=0.004, \eta_{\mathrm{p} 2}=0.337$ ) on N2. The painful picture elicited a more positive N2 than the non-painful pictures $(-0.738 \pm 0.119 \mu \mathrm{V}$ and $-0.993 \pm 0.156 \mu \mathrm{V})$. The main effect of Context $\left(\mathrm{F}(1,21)=1.539, p=0.228, \eta_{\mathrm{p} 2}=0.068\right)$ and the interaction of Context $\times$ Picture $(\mathrm{F}(1,21)=0.024, p=0.878$, $\left.\eta_{\mathrm{p} 2}=0.001\right)$ were insignificant.

P3 The main effect of Picture $(\mathrm{F}(1,21)=1.995, p=0.172$, $\left.\eta_{\mathrm{p} 2}=0.087\right)$ and Context $\left(\mathrm{F}(1,21)=0.247, p=0.625, \eta_{\mathrm{p}}{ }^{2}=\right.$ 0.012 ) were not significant. We found a significant interaction of Context $\times$ Picture $\left(\mathrm{F}(1,21)=5.433, p=0.030, \eta_{\mathrm{p} 2}=\right.$ 0.206). Pairwise comparisons showed that only in the friendly context did the painful pictures elicit a significantly larger amplitude than the non-painful pictures $(2.650 \pm 0.215 \mu \mathrm{V}$ and $2.009 \pm 0.167 \mu \mathrm{V}, p=0.031)$. Meanwhile no significant difference was observed between the painful and the non- 


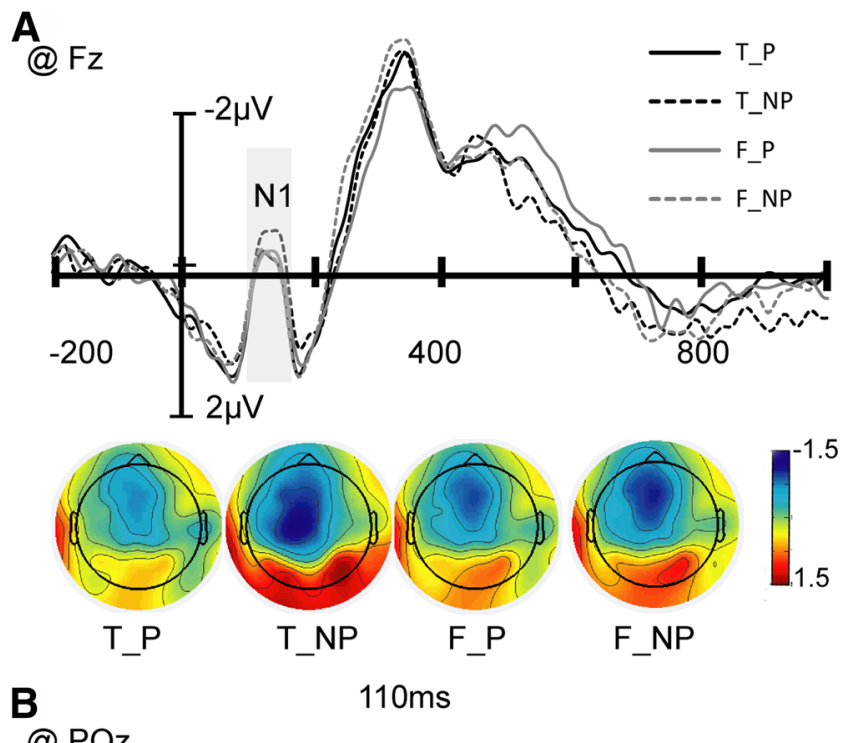

@ POz

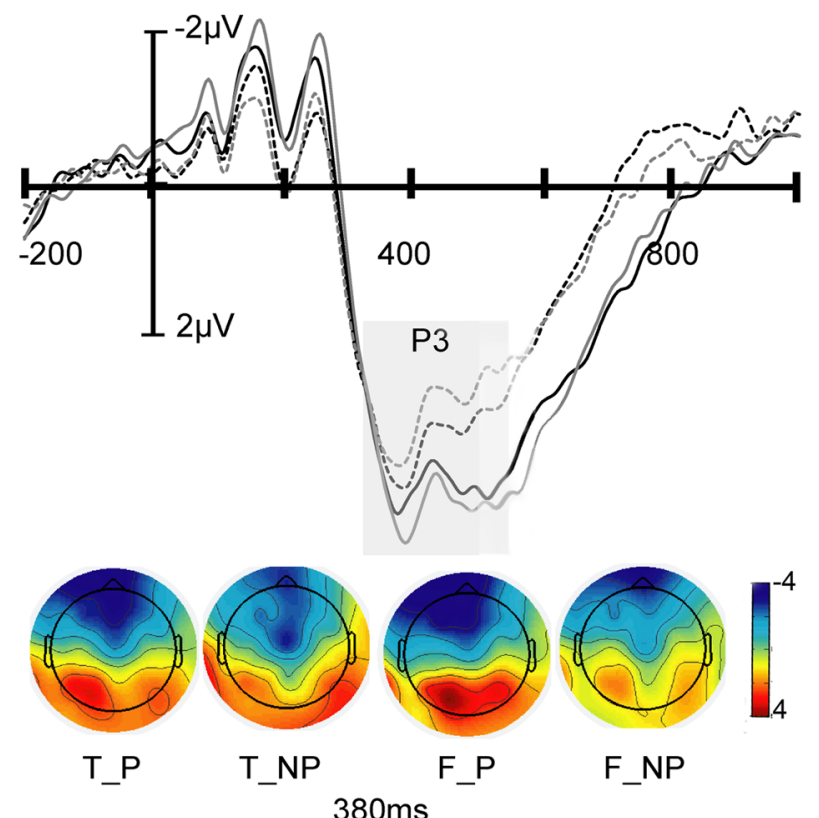

Fig. 2 (A) Grand average and topography of N1 at Fz site; (B) grand average and topography of $\mathrm{P} 3$ at $\mathrm{POz}$ site under four conditions: the painful picture in the threatening context (T_P); the non-painful pictures in the threatening context (T_NP); the painful picture in the friendly context (F_P); and the non-painful pictures in the friendly context (F_NP) painful pictures in the threatening context pictures $(2.418 \pm$ $0.223 \mu \mathrm{V}$ and $1.897 \pm 0.156 \mu \mathrm{V}, p=0.760$ ) (Fig. $2 \mathrm{~B}$ and Fig. 3). The figure of ERPs waveforms with the continuous SD is provided in the Supplementary Material, Fig. S1. No other significant effects or interactions were observed.

\section{Discussion}

In the present study, ERP responses to viewing others' pain in a threatening context and a friendly context were compared. Consistent with previous findings (Decety et al., 2010; Fan \& Han, 2008; Meng et al., 2012), early automatic components (N1 and N2) in the frontal area and later positive deflections (P3) over the posterior parietal area were observed. A significant Context $\times$ Picture interaction was found, in which the painful pictures elicited a more positive amplitude of N1 than the non-painful pictures only in the threatening context and not in the friendly context. An inverse Context $\times$ Picture interaction was also found, in which the painful pictures elicited a larger amplitude of $\mathrm{P} 3$ than the non-painful pictures only in the friendly context and not in the threatening context.

The literature indicates that $\mathrm{N} 1$ can discriminate painful from non-painful stimuli and may be considered a marker of the automatic activation of affective arousal or emotional sharing (Decety, 2011; Lyu et al., 2014). In the present study, we found a more positive shift of $\mathrm{N} 1$ in response to painful stimuli compared with non-painful stimuli in the threatening context, but no such effect was found in the friendly context. This effect may be better explained by the TVPH, which proposes that observing others' pain may activate the threat-detection system rather than trigger an automatic empathic response. The threat-detection system would be more alert in a threatening context than in a safe context, and the noxious and neutral stimuli would be better differentiated in a threatening context accordingly. One ERP study compared self-inflicted pain and pain that was caused by others and reported a similar effect, in which painful stimuli elicited a more positive N1 than non-painful stimuli only when the individual who was the target of pain was with others and not when the target was alone (Lyu et al., 2014). These findings can also be explained
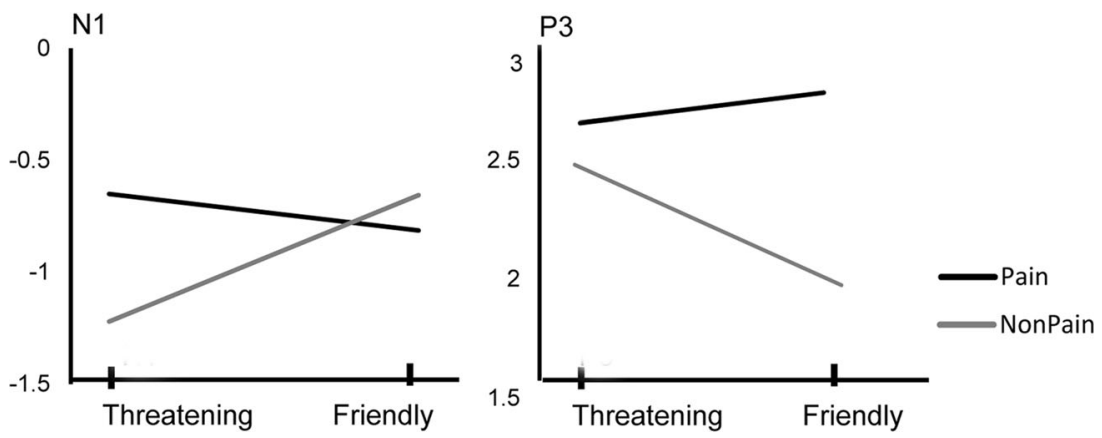

Fig. 3 The interaction effect observed on the N1 and P3 components 
by the TVPH. When another person was with the target individual in the picture, the context was considered more threatening and dangerous than when the target was alone (Ibanez et al., 2011). Another ERP study found that differences in the effects of painful and non-painful stimuli on the amplitudes of N1 were significant in a group of adolescents but nonsignificant in a group of adults (Mella et al., 2012). This result might also support the TVPH because adolescents have been reported to be more sensitive to threats and dangers than adults (Yuan et al., 2015).

The stage of early perceptual processing of others' pain would more likely be a threat-detection stage instead of an empathizing stage. This makes sense from an evolutionary perspective. Whenever a noxious stimulus appears, the brain quickly detects whether it is dangerous. When we see others' pain, we first immediately decide whether this pain is a danger warning to us rather than empathize with it. For example, the sight of a wounded person after hearing a gunshot may indicate the presence of a killer. Your first reaction would be, "I should run for my life!" instead of "Ouch! That must hurt!"

One study measured local field potentials in patients with depth electrodes placed in the amygdala and several other regions while they observed pictures that showed intentional and unintentional harm. Intentional harm induced activity in the amygdala in a very early stage $(<200 \mathrm{~ms}$; Hesse et al., 2016). This finding indicates that the detection of intentional harm occurs very early, and the amygdala is a critical hub to index the biological significance of salient stimuli (Hesse et al., 2016; Pessoa \& Adolphs, 2010). In the present study, the threatening context might activate the amygdala in the observer because intentional harm is more likely to occur in a threatening context than in a friendly context. The activated danger detection system enhances the sensitivity to noxious stimuli and facilitates the subsequent categorization of harmful events (Pessoa \& Adolphs, 2010). The neural underpinnings of the effect of N1 might involve the amygdala, and its frontotemporal connections may trigger an early response to harmful scenarios only in a threatening context and not in a friendly context.

We found a significant effect of picture on N2, which was larger in response to painful stimuli compared with non-painful stimuli, indicating that this component can discriminate painful stimuli from non-painful stimuli. The involvement of the $\mathrm{N} 2$ component in observing others' physical pain has been consistently reported, and it was suggested to be an index of an early automatic component that is related to the sensitivity to others' pain and a biomarker of the affective component of empathy for pain (Chen, Yang, \& Cheng, 2012). In the present study, the amplitude of $\mathrm{N} 2$ was not modulated by the social context, which may indicate that $\mathrm{N} 2$ was a relatively stable biomarker of the sensitivity to others' pain.
Previous studies suggested that affective pictures elicited a larger P3 than neutral pictures, and P3 was suggested to reflect motivational engagement and the commitment of attentional resources to affective pictures (Olofsson, Nordin, Sequeira, \& Polich, 2008; Schupp et al., 2000). Stimuli that are more salient, arousing, and motivationally significant usually elicit a larger P3 (Delplanque, Lavoie, Hot, Silvert, \& Sequeira, 2004; Keil et al., 2002; Mini, Palomba, Angrilli, \& Bravi, 1996; Nieuwenhuis, Aston-Jones, \& Cohen, 2005; Sabatinelli, Lang, Keil, \& Bradley, 2007). In the present study, the painful pictures elicited a significantly larger $\mathrm{P} 3$ than non-painful pictures in the friendly context but not in the threatening context. This result appears to be consistent with the "empathizing hypothesis." The friendly context promotes an empathic response. Previous studies found a moderately significant relationship between positive affect and empathy, in which people exhibited higher levels of empathy when they were in a positive mood (Likowski, Muhlberger, Seibt, Pauli, \& Weyers, 2011; Rehberger, 2014). In the present study, the friendly context (i.e., observing a series of smiling faces) may have induced a positive mood in the participants, thus increasing their empathy for others' pain. A threatening context may suppress the empathic response to others' pain. One study supported this assumption by finding that $\mathrm{P} 3$ amplitudes in response to observing others' pain significantly decreased when self-pain primes rather than neutral primes were presented (Meng et al., 2013). Another recent study found that the stress of being around strangers reduced empathic responses to others' pain (Martin et al., 2015). In a friendly context, people would be more likely to allocate mental resources to processing others' feelings because their own safety is not threatened. Therefore, we suggest that during the later, cognitive processing stage, observing others' pain triggers an empathic response, as proposed by the "empathizing hypothesis."

Notably, in the present study, an affective oddball paradigm was used. The effect on P3 that we observed could be explained by an oddball effect rather than by modulation of the empathic response by the social context. Studies that have used the affective oddball paradigm found that a greater affective distance from the target (i.e., a greater difference in valence from the standard stimuli) was associated with a larger P3 (Cacioppo \& Berntson, 1994; Schupp et al., 2000;). When the affective valence of the deviant stimuli are inconsistent with the valence of the standard stimuli, this affective inconsistency elicits a substantially larger $\mathrm{P} 3$, which is conceptualized to be attributable to a mismatch between the affective context and affective evaluation of the stimuli (Cacioppo, Crites, Jr., \& Coles, 1993; Crites, Cacioppo, Gardner, \& Berntson, 1995). In the present study, the distance in valence between the affective context (smiling faces) and painful stimuli in the friendly context was larger than the distance in valence between the affective context (angry faces) and painful stimuli in the threatening context. Thus, the greater contrast in 
the friendly context may explain the observed effect on P3. The literature on empathy for pain has consistently indicated that P3 reflects an empathic response. Studies that have used the affective oddball paradigm have also indicated that the apparent probability of stimuli and the intrinsic affective properties of stimuli modulate P3. Therefore, we cannot distinguish the oddball effect from the empathic effect based on the present data. We suggest that the effect on P3 may have been partially attributable to an oddball effect. Future studies should control the probabilities of stimuli to clarify the "pure" contextual modulation of empathic responses on P3.

One issue is why the same stimulus (i.e., others' pain) triggers a different neural response in different contexts. The social context network model (SCNM), proposed by Ibanez, suggests that in social situations, people use common sense and implicit associative learning that derives from prior experience to update the contextual framework to predict the meaning of social targets that are most likely to appear in a specific scene (Melloni et al., 2014). For example, in a threatening context, based on prior experience, observers would predict the meaning of others' pain as a warning of imminent danger. Therefore, observers would recruit most of their mental resources to protect themselves. In a friendly context, observers would predict the same signal of others' pain as a call for help. Without the stress of being threatened, they tend to empathize with others' pain. What is the neural basis for this contextual modulation? The SCNM suggests that a frontotemporo-insular network mediates such modulation (Ibanez $\&$ Manes, 2012). The social context network modulates and influences the pain matrix, depending on the specificity of the situation and saliency of the event, allowing a very complex pool of empathic responses for each situation (Melloni et al., 2014). The present findings support the SCNM by showing that others' pain can trigger different automatic and controlled neural responses in different social contexts. Furthermore, the "empathizing hypothesis" and TVPH may hold true in different temporal processing stages. The theoretical framework of the SCNM may explain which of these two hypotheses predominates and why in specific social contexts. However, the ways in which the interaction between empathy for pain and social context networks impact this modulation require further investigation, especially with functional magnetic resonance imaging studies.

The present findings should be considered heuristic rather than prescriptive. We provide temporal proof that the social context can modulate the perception of others' pain. The two different hypotheses ("empathizing hypothesis" and TVPH) might hold true in different processing stages instead of being mutually exclusive. However, we were unable to separate the odd-ball effect from the context effect on P3 because of limitations that are inherent with the experimental design. We were also unable to uncover the neural underpinnings of such an effect observed due to the limitation of the technology applied. Future studies should broaden the experimental design and perform analyses with higher spatial resolution.

In summary, the present results indicate that the social context modulates the neural responses to perceiving others' pain. Our findings suggest that the "empathizing hypothesis" and TVPH are not mutually exclusive and might work in different processing stages. The early, automatic processing stage was better explained as a danger-detection process, and the later, cognitive processing stage was better explained as an empathizing process. The present results should be further explored based on the theoretical framework of the SCNM.

Acknowledgements This study was funded by the National Natural Science Foundation of China, no. 31500877 and 31300846. We thank Jing Meng for sending us the pictures we used in the experiment (Meng et al., 2013).

\section{Compliance with ethical standards}

Conflict of interest statement The authors declare that they have no conflicts of interest that are relevant to this study.

\section{References}

Akitsuki, Y., \& Decety, J. (2009). Social context and perceived agency affects empathy for pain: an event-related fMRI investigation. NeuroImage, 47, 722-734.

Barnett, E., \& Casper, M. (2001). A definition of "social environment". American Journal of Public Health, 91, 465.

Cacioppo, J. T. \& Berntson, G. G. (1994). Relationships between attitudes and evaluative space: A critical review, with emphasis on the separability of positive and negative substrates. Psychological Bulletin, 401-423.

Cacioppo, J. T., Crites, S. L., Jr., Berntson, G. G., \& Coles, M. G. H. (1993). If attitudes affect how stimuli are processed, should they not affect the event-related brain potential? Psychological Science, 4, $108-113$.

Chen, C., Yang, C. Y., \& Cheng, Y. (2012). Sensorimotor resonance is an outcome but not a platform to anticipating harm to others. Social Neuroscience, 7, 578-590.

Crites, S. L., Cacioppo, J. T., Gardner, W. L., \& Berntson, G. G. (1995). Bioelectrical echoes from evaluative categorization: II. A late positive brain potential that varies as a function of attitude registration rather than attitude report. Journal of Personality and Social Psychology, 68, 997-1013.

Cui, F., Ma, N., \& Luo, Y. J. (2016). Moral judgment modulates neural responses to the perception of other's pain: An ERP study. Scientific Reports, 6, 20851.

Cui, F., Zhu, X., Duan, F., \& Luo, Y. (2015). Instructions of cooperation and competition influence the neural responses to others' pain: An ERP study. Social Neuroscience, 1-8.

Cui, F., Zhu, X., Gu, R., \& Luo, Y. J. (2016). When your pain signifies my gain: neural activity while evaluating outcomes based on another person's pain. Scientific Reports, 6, 26426.

Decety, J. (2011). Dissecting the neural mechanisms mediating empathy. Emotion Review, 2, 92-108.

Decety, J., Echols, S., \& Correll, J. (2010). The blame game: The effect of responsibility and social stigma on empathy for pain. Journal of Cognitive Neuroscience, 22, 985-997. 
Decety, J., \& Jackson, P. L. (2004). The functional architecture of human empathy. Behavioral and Cognitive Neuroscience Reviews, 3, 71100

Decety, J., \& Lamm, C. (2006). Human empathy through the lens of social neuroscience. ScientificWorldJournal, 6, 1146-1163.

Decety, J., Yang, C. Y., \& Cheng, Y. (2010). Physicians down-regulate their pain empathy response: An event-related brain potential study. NeuroImage, 50, 1676-1682.

Delorme, A., \& Makeig, S. (2004). EEGLAB: An open source toolbox for analysis of single-trial EEG dynamics including independent component analysis. Journal of Neuroscience Methods, 134, 9-21.

Delplanque, S., Lavoie, M. E., Hot, P., Silvert, L., \& Sequeira, H. (2004). Modulation of cognitive processing by emotional valence studied through event-related potentials in humans. Neuroscience Letters, $356,1-4$.

Fan, Y., \& Han, S. (2008). Temporal dynamic of neural mechanisms involved in empathy for pain: An event-related brain potential study. Neuropsychologia, 46, 160-173.

Gong, X., Huang, Y., Wang, Y., \& Luo, Y. (2011). Revision of the Chinese facial affective picture system. Chinese Mental Health Journal, 25, 40-46.

Gu, X., \& Han, S. (2007). Attention and reality constraints on the neural processes of empathy for pain. NeuroImage, 36, 256-267.

Han, S., Fan, Y., \& Mao, L. (2008). Gender difference in empathy for pain: An electrophysiological investigation. Brain Research, 1196, 85-93.

Hesse, E., Mikulan, E., Decety, J., Sigman, M., Garcia, M. C., Silva, W., Ciraolo,C., Vaucheret, E., Baglivo.F, Huepe, D. Lopez, V., Manes, F., Bekinschtein, T. A., Ibanez, A. (2016). Early detection of intentional harm in the human amygdala. Brain, 139, 54-61.

Ibanez, A., Hurtado, E., Lobos, A., Escobar, J., Trujillo, N., Baez, S., Huepe, D., Manes, F., Decety, J. (2011). Subliminal presentation of other faces (but not own face) primes behavioral and evoked cortical processing of empathy for pain. Brain Research, 1398, $72-85$.

Ibanez, A., \& Manes, F. (2012). Contextual social cognition and the behavioral variant of frontotemporal dementia. Neurology, 78, 1354-1362.

Jung, T. P., Makeig, S., Westerfield, M., Townsend, J., Courchesne, E., \& Sejnowski, T. J. (2001). Analysis and visualization of single-trial event-related potentials. Human Brain Mapping, 14, 166-185.

Karos, K., Meulders, A., \& Vlaeyen, J. W. (2015). Threatening social context facilitates pain-related fear learning. The Journal of Pain, 16, 214-225.

Keil, A., Bradley, M. M., Hauk, O., Rockstroh, B., Elbert, T., \& Lang, P. J. (2002). Large-scale neural correlates of affective picture processing. Psychophysiology, 39, 641-649.

Keysers, C., Kaas, J. H., \& Gazzola, V. (2010). Somatosensation in social perception. Nature Reviews. Neuroscience, 11, 417-428.

Keysers, C., Meffert, H., \& Gazzola, V. (2014). Reply: Spontaneous versus deliberate vicarious representations: different routes to empathy in psychopathy and autism. Brain.

Lamm, C., Decety, J., \& Singer, T. (2011). Meta-analytic evidence for common and distinct neural networks associated with directly experienced pain and empathy for pain. NeuroImage, 54, 2492-2502.
Likowski, K. U., Muhlberger, A., Seibt, B., Pauli, P., \& Weyers, P. (2011). Processes underlying congruent and incongruent facial reactions to emotional facial expressions. Emotion, 11, 457-467.

Lyu, Z., Meng, J., \& Jackson, T. (2014). Effects of cause of pain on the processing of pain in others: An ERP study. Experimental Brain Research, 232, 2731-2739.

Martin, L. J., Hathaway, G., Isbester, K., Mirali, S., Acland, E. L., Niederstrasser, N., Slepian, P.M., Trost, Z., Bartz, J. A., Sapolsky, R. M., Sternberg, W. F., Levitin, D. J., Mogil, J. S. (2015). Reducing social stress elicits emotional contagion of pain in mouse and human strangers. Current Biology, 25, 326-332.

Mella, N., Studer, J., Gilet, A. L., \& Labouvie-Vief, G. (2012). Empathy for pain from adolescence through adulthood: An event-related brain potential study. Frontiers in Psychology, 3, 501.

Melloni, M., Lopez, V., \& Ibanez, A. (2014). Empathy and contextual social cognition. Cognitive, Affective, \& Behavioral Neuroscience, $14,407-425$.

Meng, J., Hu, L., Shen, L., Yang, Z., Chen, H., Huang, X., Jackson, T. (2012). Emotional primes modulate the responses to others' pain: an ERP study. Experimental Brain Research, 220, 277-286.

Meng, J., Jackson, T., Chen, H., Hu, L., Yang, Z., Su, Y., Huang, X. (2013). Pain perception in the self and observation of others: An ERP investigation. NeuroImage, 72, 164-173.

Mini, A., Palomba, D., Angrilli, A., \& Bravi, S. (1996). Emotional information processing and visual evoked brain potentials. Perceptual and Motor Skills, 83, 143-152.

Nieuwenhuis, S., Aston-Jones, G., \& Cohen, J. D. (2005). Decision making, the P3, and the locus coeruleus-norepinephrine system. Psychological Bulletin, 131, 510-532.

Olofsson, J. K., Nordin, S., Sequeira, H., \& Polich, J. (2008). Affective picture processing: An integrative review of ERP findings. Biological Psychology, 77, 247-265.

Pessoa, L., \& Adolphs, R. (2010). Emotion processing and the amygdala: From a 'low road' to 'many roads' of evaluating biological significance. Nature Review Neuroscience, 11, 773-783.

Rehberger, C. (2014). Examining the relationships between empathy, mood, and facial mimicry. DePaul Discoveries, 3.

Rizzolatti, G., \& Sinigaglia, C. (2010). The functional role of the parietofrontal mirror circuit: Interpretations and misinterpretations. Nature Reviews. Neuroscience, 11, 264-274.

Sabatinelli, D., Lang, P. J., Keil, A., \& Bradley, M. M. (2007). Emotional perception: Correlation of functional MRI and event-related potentials. Cerebral Cortex, 17, 1085-1091.

Schupp, H. T., Cuthbert, B. N., Bradley, M. M., Cacioppo, J. T., Ito, T., \& Lang, P. J. (2000). Affective picture processing: The late positive potential is modulated by motivational relevance. Psychophysiology, 37, 257-261.

Sessa, P., Meconi, F., Castelli, L., \& Dell'Acqua, R. (2014). Taking one's time in feeling other-race pain: An event-related potential investigation on the time-course of cross-racial empathy. Social Cognitive and Affective Neuroscience, 9, 454-463.

Singer, T., Seymour, B., O'Doherty, J., Kaube, H., Dolan, R. J., \& Frith, C. D. (2004). Empathy for pain involves the affective but not sensory components of pain. Science, 303, 1157-1162.

Yuan, J., Ju, E., Meng, X., Chen, X., Zhu, S., Yang, J., Li, H. (2015). Enhanced brain susceptibility to negative stimuli in adolescents: ERP evidences. Frontiers in Behavioral Neuroscience, 9, 98. 